\title{
ON CERTAIN NONLINEAR INTEGRAL INEQUALITIES INVOLVING ITERATED INTEGRALS
}

\author{
B. G. PACHPATTE
}

\begin{abstract}
In this paper explicit bounds on some nonlinear integral inequalities involving iterated integrals are established. Applications are also given to illustriate the usefulness of one of our results.
\end{abstract}

\section{Introduction}

Integral inequalities with iterated integrals play a very important role in the study of various classes of integrodifferential and integral equations. In [3] Bykov and Salpagarov (see also [1, 4-6]) have given the explicit bounds on the following integral inequalities

$$
\begin{aligned}
u(t) \leq & +\int_{\alpha}^{t} b(s) u(s) d s+\int_{\alpha}^{t}\left(\int_{\alpha}^{s} k(s, \tau) u(\tau) d \tau\right) d s \\
& +\int_{\alpha}^{t}\left(\int_{\alpha}^{s}\left(\int_{\alpha}^{\tau} h(s, \tau, \sigma) u(\sigma) d \sigma\right) d \tau\right) d s, \\
u(t) \leq c & +\int_{\alpha}^{t} k(t, s) u(s) d s+\int_{\alpha}^{t}\left(\int_{\alpha}^{s} h(t, s, \sigma) u(\sigma) d \sigma\right) d s
\end{aligned}
$$

under some suitable conditions on the functions involved in (1.1) and (1.2). For a detailed account on such inequalities and their applications, see [1-7]. Motivated by the results given in [3], in this paper we offer some useful nonlinear generalizations of the inequalities in [3] which will be equally important to achieve a diversity of desired goals in certain applications. The two independent variable generalizations of the main results and some applications of one of our results are also given.

\section{Statement of Results}

Let $R$ denotes the set of real numbers; $R_{+}=[0, \infty), I=[0, T), I_{1}=[0, X), I_{2}=[0, Y)$ are the given subsets of $R, \Delta=I_{1} \times I_{2}$ and ' denotes the derivative. The partial

Received August 9, 2004.

2000 Mathematics Subject Classification. 26D10, 26D15.

Key words and phrases. Nonlinear integral inequalities, iterated integrals, explicit bounds, partial derivatives, Volterra integral equation. 
derivatives of a function $z(x, y)$ for $x, y \in R$ with respect to $x, y$ and $x y$ are denoted by $D_{1} z(x, y), D_{2} z(x, y)$ and $D_{1} D_{2} z(x, y)=D_{2} D_{1} z(x, y)$ (or $\frac{\partial}{\partial x} z(x, y), \frac{\partial}{\partial y} z(x, y)$ and $\left.\frac{\partial^{2}}{\partial x \partial y} z(x, y)=\frac{\partial^{2}}{\partial y \partial x} z(x, y)\right)$ respectively. We denote by $E_{1}=\left\{(t, s) \in I^{2}: 0 \leq s \leq t<T\right\}$, $E_{2}=\left\{(t, s, \sigma) \in I^{3}: 0 \leq \sigma \leq s \leq t<T\right\}, H_{1}=\left\{(x, y, s, t) \in \Delta^{2}: 0 \leq s \leq x<X, 0 \leq\right.$ $t \leq y<Y\}, H_{2}=\left\{(x, y, s, t, \sigma, \tau) \in \Delta^{3}: 0 \leq \sigma \leq s \leq x<X, 0 \leq \tau \leq t \leq y<Y\right\}$. Throughout, we assume that all the integrals involved in the discussion exist on the respective domains of their definitions and are finite.

Our main results are given in the following theorem.

Theorem 1. Let $u(t) \in C\left(I, R_{+}\right), k(t, s) \in C\left(E_{1}, R_{+}\right), h(t, s, \sigma) \in C\left(E_{2}, R_{+}\right)$and $a(t), a^{\prime}(t) \in C\left(I, R_{+}\right)$. Let $g(u) \in C\left(R_{+}, R_{+}\right)$be a nondecreasing function, $g(u)>0$ on $(0, \infty)$.

$\left(c_{1}\right)$ Let $b(t) \in C\left(I, R_{+}\right)$. If

$$
\begin{aligned}
u(t) \leq & a(t)+\int_{0}^{t} b(s) g(u(s)) d s+\int_{0}^{t}\left(\int_{0}^{s} k(s, \tau) g(u(\tau)) d \tau\right) d s \\
& +\int_{0}^{t}\left(\int_{0}^{s}\left(\int_{0}^{\tau} h(s, \tau, \sigma) g(u(\sigma)) d \sigma\right) d \tau\right) d s,
\end{aligned}
$$

for $t \in I$, then for $0 \leq t \leq t_{1} ; t, t_{1} \in I$.

$$
u(t) \leq G^{-1}\left[G(a(t))+\int_{0}^{t} M(s) d s\right]
$$

where

$$
G(r)=\int_{r_{0}}^{r} \frac{d s}{g(s)}, \quad r>0,
$$

$r_{0}>0$ is arbitrary, $G^{-1}$ is the inverse of $G$,

$$
M(t)=b(t)+\int_{0}^{t} k(t, \tau) d \tau+\int_{0}^{t}\left(\int_{0}^{\tau} h(t, \tau, \sigma) d \sigma\right) d \tau
$$

for $t \in I$ and $t_{1} \in I$ is chosen so that

$$
G(a(t))+\int_{0}^{t} M(s) d s \in \operatorname{Dom}\left(G^{-1}\right)
$$

for all $t \in I$ lying in the interval $0 \leq t \leq t_{1}$.

$\left(c_{2}\right)$ Let $\frac{\partial}{\partial t} k(t, x) \in C\left(E_{1}, R_{+}\right), \frac{\partial}{\partial t} h(t, s, \sigma) \in C\left(E_{2}, R_{+}\right)$. If

$$
u(t) \leq a(t)+\int_{0}^{t} k(t, s) g(u(s)) d s+\int_{0}^{t}\left(\int_{0}^{s} h(t, s, \sigma) g(u(\sigma)) d \sigma\right) d s,
$$


for $t \in I$, then for $0 \leq t \leq t_{2} ; t, t_{2} \in I$,

$$
u(t) \leq G^{-1}\left[G(a(t))+\int_{0}^{t}[R(s)+Q(s)] d s\right],
$$

where $G, G^{-1}$ are as defined in part $\left(c_{1}\right)$,

$$
\begin{aligned}
& R(t)=k(t, t)+\int_{0}^{t} h(t, t, \sigma) d \sigma, \\
& Q(t)=\int_{0}^{t} \frac{\partial}{\partial t} k(t, \sigma) d \sigma+\int_{0}^{t}\left(\int_{0}^{s} \frac{\partial}{\partial t} h(t, s, \sigma) d \sigma\right) d s,
\end{aligned}
$$

for $t \in I$ and $t_{2} \in I$ is chosen so that

$$
G(a(t))+\int_{0}^{t}[R(s)+Q(s)] d s \in \operatorname{Dom}\left(G^{-1}\right),
$$

for all $t \in I$ lying in the interval $0 \leq t \leq t_{2}$.

Remark 1. If we take $g(u)=u$, then $G(r)=\log \frac{r}{r_{0}}, G^{-1}(r)=r_{0} \exp (r)$ and the bounds obtained in (2.2) and (2.6) reduces respectively to

$$
u(t) \leq a(t) \exp \left(\int_{0}^{t} M(s) d s\right)
$$

and

$$
u(t) \leq a(t) \exp \left(\int_{0}^{t}[R(s)+Q(s)] d s\right),
$$

for $t \in I$. Furthermore, if we take $a(t)=c$, a nonnegative constant, then we get the inequalities established by Bykov and Salpagarov in [3] (see also [1, 4-6]).

The following theorem deals with the two independent variable versions of the inequalities established in Theorem 1 which can be used in certain applications.

Theorem 2. Let $u(x, y) \in C\left(\Delta, R_{+}\right), k(x, y, s, t) \in C\left(H_{1}, R_{+}\right), h(x, y, s, t, \sigma, \tau) \in$ $C\left(H_{2}, R_{+}\right)$and $a(x, y), D_{1} a(x, y), D_{2} a(x, y), D_{1} D_{2} a(x, y) \in C\left(\Delta, R_{+}\right)$. Let $g(u)$ be $a$ continuously differentiable function defined for $u \geq 0, g(u)>0$ for $u>0$ and $g^{\prime}(u) \geq 0$ for $u \geq 0$.

$\left(d_{1}\right)$ Let $b(x, y) \in C\left(\Delta, R_{+}\right)$. If

$$
\begin{aligned}
u(x, y) \leq & a(x, y)+\int_{0}^{x} \int_{0}^{y} b(s, t) g(u(s, t)) d t d s \\
& +\int_{0}^{x} \int_{0}^{y}\left(\int_{0}^{s} \int_{0}^{t} k(s, t, \sigma, \tau) g(u(\sigma, \tau)) d \tau d \sigma\right) d t d s \\
& +\int_{0}^{x} \int_{0}^{y}\left(\int_{0}^{s} \int_{0}^{t}\left(\int_{0}^{\sigma} \int_{0}^{\tau} h(s, t, \sigma, \tau, m, n) g(u(m, n)) d n d m\right) d \tau d \sigma\right) d t d s
\end{aligned}
$$


for $(x, y) \in \Delta$, then for $0 \leq x \leq x_{1}, 0 \leq y \leq y_{1} ; x, x_{1} \in I_{1}, y, y_{1} \in I_{2}$,

$$
u(x, y) \leq G^{-1}\left[G(a(x, y))+\int_{0}^{x} \int_{0}^{y} N(s, t) d t d s\right]
$$

where

$$
\begin{aligned}
N(x, y)= & b(x, y)+\int_{0}^{x} \int_{0}^{y} k(x, y, \sigma, \tau) d \tau d \sigma \\
& +\int_{0}^{x} \int_{0}^{y}\left(\int_{0}^{\sigma} \int_{0}^{\tau} h(x, y, \sigma, \tau, m, n) d n d m\right) d \tau d \sigma,
\end{aligned}
$$

$G, G^{-1}$ are as in Theorem 1 part $\left(c_{1}\right)$ and $x_{1} \in I_{1}, y_{1} \in I_{2}$ are chosen so that

$$
G(a(x, y))+\int_{0}^{x} \int_{0}^{y} N(s, t) d t d s \in \operatorname{Dom}\left(G^{-1}\right),
$$

for all $(x, y) \in \Delta$ such that $0 \leq x \leq x_{1}, 0 \leq y \leq y_{1}$.

$\left(d_{2}\right)$ Let $D_{1} k(x, y, s, t), D_{2} k(x, y, s, t), D_{2} D_{1} k(x, y, s, t) \in C\left(H_{1}, R_{+}\right) ; D_{1} h(x, y, s, t, \sigma, \tau)$, $D_{2} h(x, y, s, t, \sigma, \tau), D_{2} D_{1} h(x, y, s, t, \sigma, \tau) \in C\left(H_{2}, R_{+}\right)$. If

$$
\begin{aligned}
u(x, y) \leq & a(x, y)+\int_{0}^{x} \int_{0}^{y} k(x, y, s, t) g(u(s, t)) d t d s \\
& +\int_{0}^{x} \int_{0}^{y}\left(\int_{0}^{s} \int_{0}^{t} h(x, y, s, t, \sigma, \tau) g(u(\sigma, \tau)) d \tau d \sigma\right) d t d s,
\end{aligned}
$$

for $(x, y) \in \Delta$, then for $0 \leq x \leq x_{2}, 0 \leq y \leq y_{2} ; x, x_{2} \in I_{1}, y, y_{2} \in I_{2}$,

$$
u(x, y) \leq G^{-1}\left[G(a(x, y))+\int_{0}^{x} \int_{0}^{y}[A(m, n)+B(m, n)] d n d m\right],
$$

where

$$
\begin{aligned}
A(x, y)= & k(x, y, x, y)+\int_{0}^{x} D_{1} k(x, y, \xi, y) d \xi+\int_{0}^{y} D_{2} k(x, y, x, \eta) d \eta \\
& +\int_{0}^{x} \int_{0}^{y} D_{2} D_{1} k(x, y, \xi, \eta) d \eta d \xi \\
B(x, y)= & \int_{0}^{x} \int_{0}^{y} h(x, y, x, y, \sigma, \tau) d \tau d \sigma+\int_{0}^{x}\left(\int_{0}^{s} \int_{0}^{y} D_{1} h(x, y, s, y, \sigma, \tau) d \tau d \sigma\right) d s \\
& +\int_{0}^{y}\left(\int_{0}^{x} \int_{0}^{t} D_{2} h(x, y, x, t, \sigma, \tau) d \tau d \sigma\right) d t \\
& +\int_{0}^{x} \int_{0}^{y}\left(\int_{0}^{s} \int_{0}^{t} D_{2} D_{1} h(x, y, s, t, \sigma, \tau) d \tau d \sigma\right) d t d s
\end{aligned}
$$

$G, G^{-1}$ are as in Theorem 1 part $\left(c_{1}\right)$ and $x_{2} \in I_{1}, y_{2} \in I_{2}$ are chosen so that

$$
G(a(x, y))+\int_{0}^{x} \int_{0}^{y}[A(m, n)+B(m, n)] d n d m \in \operatorname{Dom}\left(G^{-1}\right),
$$

for all $(x, y) \in \Delta$ such that $0 \leq x \leq x_{2}, 0 \leq y \leq y_{2}$. 
Remark 2. By taking $g(u)=u$ in Theorem 2, it is easy to observe that the bounds in (2.12) and (2.15) reduces respectively to

$$
u(x, y) \leq a(x, y) \exp \left(\int_{0}^{x} \int_{0}^{y} N(s, t) d t d s\right)
$$

and

$$
u(x, y) \leq a(x, y) \exp \left(\int_{0}^{x} \int_{0}^{y}[A(m, n)+B(m, n)] d n d m\right),
$$

for $(x, y) \in \Delta$. In this case, the inequalities obtained in (2.18) and (2.19) can be considered as generalizations of the Wendroff's inequality given in [2, p.154]. For a large number of such inequalities and their applications, we refer the interested readers to $[1$, $7]$.

\section{Proof of Theorem 1}

First we note that, since $a^{\prime}(t) \geq 0$, the function $a(t)$ is monotonically increasing (see $[8, \mathrm{p} .81])$.

$\left(c_{1}\right)$ Let $a(t)>0$ for $t \in I$ and define a function $z(t)$ by the right hand side of (2.1). Then $z(t)>0, z(0)=a(0), u(t) \leq z(t)$ and by hypotheses, it is nondecreasing and

$$
\begin{aligned}
z^{\prime}(t) & =a^{\prime}(t)+b(t) g(u(t))+\int_{0}^{t} k(t, \tau) g(u(\tau)) d \tau+\int_{0}^{t}\left(\int_{0}^{\tau} h(t, \tau, \sigma) g(u(\sigma)) d \sigma\right) d \tau \\
& \leq a^{\prime}(t)+b(t) g(z(t))+\int_{0}^{t} k(t, \tau) g(z(\tau)) d \tau+\int_{0}^{t}\left(\int_{0}^{\tau} h(t, \tau, \sigma) g(z(\sigma)) d \sigma\right) d \tau \\
& \leq a^{\prime}(t)+M(t) g(z(t)) .
\end{aligned}
$$

From $(2.3),(3.1)$, the fact that $a(t) \leq z(t)$ and the nondecreasing character of $g$ we have

$$
\begin{aligned}
& \frac{d}{d t} G(z(t))=\frac{z^{\prime}(t)}{g(z(t))} \\
\leq & \frac{a^{\prime}(t)+M(t) g(z(t))}{g(z(t))} \\
\leq & \frac{a^{\prime}(t)}{g(a(t))}+M(t) \\
= & \frac{d}{d t} G(a(t))+M(t) .
\end{aligned}
$$

By setting $t=s$ in (3.2) and integrating it from 0 to $t, t \in I$ we have

$$
G(z(t)) \leq G(a(t))+\int_{0}^{t} M(s) d s .
$$


From (3.3) and the hypotheses on $G$ we have

$$
z(t) \leq G^{-1}\left[G(a(t))+\int_{0}^{t} M(s) d s\right] .
$$

Using (3.4) in $u(t) \leq z(t)$ we get the required inequality in (2.2). If $a(t)$ is nonnegative, we carry out the above procedure with $a(t)+\varepsilon$ instead of $a(t)$, where $\varepsilon>0$ is an arbitrary small constant, and subsequently pass to the limit $\varepsilon \rightarrow 0$ to obtain (2.2). The subinterval $0 \leq t \leq t_{1}$ is obvious.

$\left(c_{2}\right)$ Let $a(t)>0$ for $t \in I$ and define a function $z(t)$ by the right hand side of (2.5). Then $z(t)>0, z(0)=a(0), u(t) \leq z(t), a(t) \leq z(t)$. In view of hypotheses, it is easy to observe that $z(t)$ is nondecreasing and

$$
\begin{aligned}
z^{\prime}(t)= & a^{\prime}(t)+k(t, t) g(u(t))+\int_{0}^{t} \frac{\partial}{\partial t} k(t, s) g(u(s)) d s \\
& +\int_{0}^{t} h(t, t, \sigma) g(u(\sigma)) d \sigma+\int_{0}^{t}\left(\int_{0}^{s} \frac{\partial}{\partial t} h(t, s, \sigma) g(u(\sigma)) d \sigma\right) d s \\
\leq & a^{\prime}(t)+k(t, t) g(z(t))+\int_{0}^{t} \frac{\partial}{\partial t} k(t, s) g(z(s)) d s \\
& +\int_{0}^{t} h(t, t, \sigma) g(z(\sigma)) d \sigma+\int_{0}^{t}\left(\int_{0}^{s} \frac{\partial}{\partial t} h(t, s, \sigma) g(z(\sigma)) d \sigma\right) d s \\
\leq & a^{\prime}(t)+[R(t)+Q(t)] g(z(t)) .
\end{aligned}
$$

The remaining proof can be completed by following the proof of part $\left(c_{1}\right)$ given above.

\section{Proof of Theorem 2}

From the hypotheses, it is easy to observe that the function $a(x, y)$ is monotonically increasing in both the variables $x$ and $y$. Furthermore, since $g^{\prime}(u) \geq 0$ on $R_{+}$, the function $g(u)$ is monotonically increasing on $(0, \infty)$ (see $[8$, p.81]).

$\left(d_{1}\right)$ Let $a(x, y)>0$ for $(x, y) \in \Delta$ and define a function $z(x, y)$ by the right hand side of (2.11). Then $z(x, y)>0$ and by hypotheses, it is nondecreasing in $(x, y) \in \Delta$, $z(x, 0)=a(x, 0), z(0, y)=a(0, y), u(x, y) \leq z(x, y)$ and

$$
\begin{aligned}
D_{1} z(x, y)= & D_{1} a(x, y)+\int_{0}^{y} b(x, t) g(u(x, t)) d t \\
& +\int_{0}^{y}\left(\int_{0}^{x} \int_{0}^{t} k(x, t, \sigma, \tau) g(u(\sigma, \tau)) d \tau d \sigma\right) d t \\
& +\int_{0}^{y}\left(\int_{0}^{x} \int_{0}^{t}\left(\int_{0}^{\sigma} \int_{0}^{\tau} h(x, t, \sigma, \tau, m, n) g(u(m, n)) d n d m\right) d \tau d \sigma\right) d t
\end{aligned}
$$




$$
\begin{aligned}
D_{2} z(x, y)= & D_{2} a(x, y)+\int_{0}^{x} b(s, y) g(u(s, y)) d s \\
& +\int_{0}^{x}\left(\int_{0}^{s} \int_{0}^{y} k(s, y, \sigma, \tau) g(u(\sigma, \tau)) d \tau d \sigma\right) d s \\
& +\int_{0}^{x}\left(\int_{0}^{s} \int_{0}^{y}\left(\int_{0}^{\sigma} \int_{0}^{\tau} h(s, y, \sigma, \tau, m, n) g(u(m, n)) d n d m\right) d \tau d \sigma\right) d s \\
D_{2} D_{1} z(x, y)= & D_{2} D_{1} a(x, y)+b(x, y) g(u(x, y))+\int_{0}^{x} \int_{0}^{y} k(x, y, \sigma, \tau) g(u(\sigma, \tau)) d \tau d \sigma \\
& +\int_{0}^{x} \int_{0}^{y}\left(\int_{0}^{\sigma} \int_{0}^{\tau} h(x, y, \sigma, \tau, m, n) g(u(m, n)) d n d m\right) d \tau d \sigma \\
\leq & D_{2} D_{1} a(x, y)+b(x, y) g(z(x, y))+\int_{0}^{x} \int_{0}^{y} k(x, y, \sigma, \tau) g(z(\sigma, \tau)) d \tau d \sigma \\
& +\int_{0}^{x} \int_{0}^{y}\left(\int_{0}^{\sigma} \int_{0}^{\tau} h(x, y, \sigma, \tau, m, n) g(z(m, n)) d n d m\right) d \tau d \sigma \\
\leq & D_{2} D_{1} a(x, y)+N(x, y) g(z(x, y)),
\end{aligned}
$$

where $N(x, y)$ is given by (2.13). It is easy to observe that

$$
D_{2} D_{1} G(z(x, y))=G^{\prime \prime}(z(x, y)) D_{1} z(x, y) D_{2} z(x, y)+G^{\prime}(z(x, y)) D_{2} D_{1} z(x, y)
$$

Since $a(x, y) \leq z(x, y), D_{1} z(x, y) \geq 0, D_{2} z(x, y) \geq 0, G^{\prime}(z(x, y))=\frac{1}{g(z(x, y))}$ and $G^{\prime \prime}(z(x, y)) \leq 0$, we obtain from (4.1) and (4.2)

$$
\begin{aligned}
D_{2} D_{1} G(z(x, y)) & \leq G^{\prime}(z(x, y)) D_{2} D_{1} z(x, y) \\
& \leq \frac{1}{g(z(x, y))}\left[D_{2} D_{1} a(x, y)+N(x, y) g(z(x, y))\right] \\
& \leq \frac{D_{2} D_{1} a(x, y)}{g(a(x, y))}+N(x, y) .
\end{aligned}
$$

On the other hand we observe that

$$
\begin{aligned}
D_{2} D_{1} G(a(x, y)) & =D_{2}\left(D_{1}\left(\int_{r_{0}}^{a(x, y)} \frac{d s}{g(s)}\right)\right) \\
& =D_{2}\left(\frac{D_{1} a(x, y)}{g(a(x, y))}\right) \\
& =\frac{g(a(x, y)) D_{2} D_{1} a(x, y)-D_{1} a(x, y) g^{\prime}(a(x, y)) D_{2} a(x, y)}{\{g(a(x, y))\}^{2}} \\
& =\frac{D_{2} D_{1} a(x, y)}{g(a(x, y))}-\frac{D_{1} a(x, y) g^{\prime}(a(x, y)) D_{2} a(x, y)}{\{g(a(s, y))\}^{2}}
\end{aligned}
$$

which implies

$$
D_{2} D_{1} G(a(x, y)) \geq \frac{D_{2} D_{1} a(x, y)}{g(a(x, y))} .
$$


From (4.3) and (4.4) we have

$$
D_{2} D_{1} G(z(x, y)) \leq D_{2} D_{1} G(a(x, y))+N(x, y)
$$

and this yields

$$
G(z(x, y)) \leq G(a(x, y))+\int_{0}^{x} \int_{0}^{y} N(s, t) d t d s
$$

which in view of the fact $u(x, y) \leq z(x, y)$ implies

$$
u(x, y) \leq G^{-1}\left[G(a(x, y))+\int_{0}^{x} \int_{0}^{y} N(s, t) d t d s\right]
$$

The case when $a(x, y) \geq 0$ follows as noted in the proof of Theorem 1 part $\left(c_{1}\right)$. The subdomain $0 \leq x \leq x_{1}, 0 \leq y \leq y_{1}$ is obvious.

$\left(d_{2}\right)$ Let $a(x, y)>0$ for $(x, y) \in \Delta$ and define a function $z(x, y)$ by the right hand side of (2.14). Then $z(x, y)>0$ and by hypotheses, it is nondecreasing in $(x, y) \in \Delta$, $z(x, 0)=a(x, 0), z(0, y)=a(0, y)$ and $u(x, y) \leq z(x, y)$. As in the proof of part $\left(d_{1}\right)$, it is easy to observe that $D_{1} z(x, y) \geq 0, D_{2} z(x, y) \geq 0$ and

$$
D_{2} D_{1} z(x, y) \leq D_{2} D_{1} a(x, y)+[A(x, y)+B(x, y)] g(z(x, y)),
$$

where $A(x, y), B(x, y)$ are given by $(2.16),(2.17)$. The rest of the proof can be completed by closely looking at the proof of part $\left(d_{1}\right)$ given above. We omit the details.

\section{Applications}

In this section, we present applications of the inequality in Theorem 1 part $\left(c_{2}\right)$ which pvovide estimates for the solutions of iterated Volterra integral equation of the form

$$
z(t)=f(t)+\int_{0}^{t} K(t, s, z(s)) d s+\int_{0}^{t}\left(\int_{0}^{s} H(t, s, \sigma, z(\sigma)) d \sigma\right) d s
$$

where $f \in C(I, R), K \in C\left(E_{1} \times R, R\right), H \in C\left(E_{2} \times R, R\right)$. Here, we note that the existence proofs for the solutions of equation (5.1) show either that the operator $T$ defined by the right hand side of equation (5.1) is a contraction (in which case one also has uniqueness) or $T$ is compact and continuous on a suitable subspace of the space of continuous functions.

Theorem 3. Suppose that the functions $f, K$ and $H$ in equation (5.1) satisfy

$$
\begin{aligned}
|f(t)| & \leq a(t), \\
|K(t, s, z)| & \leq k(t, s) g(|z|), \\
|H(t, s, \sigma, z)| & \leq h(t, s, \sigma) g(|z|),
\end{aligned}
$$


where $a, k, h, g$ are as in Theorem 1 part $\left(c_{2}\right)$. If $z(t), t \in I$ is any solution of equation (5.1), then

$$
|z(t)| \leq G^{-1}\left[G(a(t))+\int_{0}^{t}[R(s)+Q(s)] d s\right],
$$

for $t \in I$, where $G, G^{-1}, R(t), Q(t)$ are as given in Theorem 1 part $\left(c_{2}\right)$.

Proof. Let $z(t)$ be a solution of equation (5.1). Using the fact that $z(t)$ is a solution of equation (5.1) and (5.2)-(5.4) we have

$$
|z(t)| \leq a(t)+\int_{0}^{t} k(t, s) g(|z(s)|) d s+\int_{0}^{t}\left(\int_{0}^{s} h(t, s, \sigma) g(|z(\sigma)|) d \sigma\right) d s .
$$

Now an application of the inequality in Theorem 1 part $\left(c_{2}\right)$ to (5.6) yields (5.5).

Theorem 4. Suppose that the functions $K, H$ and $f$ in equation (5.1) satisfy

$$
\begin{gathered}
|K(t, s, z)-K(t, s, \bar{z})| \leq k(t, x) g(|z-\bar{z}|), \\
|H(t, s, \sigma, z)-H(t, s, \sigma, \bar{z})| \leq h(t, s, \sigma) g(|z-\bar{z}|), \\
\int_{0}^{t}|K(t, s, f(s))| d s+\int_{0}^{t}\left(\int_{0}^{s}|H(t, s, \sigma, f(\sigma))| d \sigma\right) d s \leq a(t),
\end{gathered}
$$

where $k, h, g, a$ are as in Theorem 1 part $\left(c_{2}\right)$. If $z(t), t \in I$ is any solution of equation (5.1), then

$$
|z(t)-f(t)| \leq G^{-1}\left[G(a(t))+\int_{0}^{t}[R(s)+Q(s)] d s\right],
$$

for $t \in I$, where $G, G^{-1}, R(s), Q(s)$ are as given in Theorem 1 part $\left(c_{2}\right)$.

Proof. Let $z(t), t \in I$ be a solution of equation (5.1). Using the fact that $z(t)$ is a solution of equation (5.1) and (5.7)-(5.9) we have

$$
\begin{aligned}
|z(t)-f(t)|= & \mid \int_{0}^{t}\{K(t, s, z(s))-K(t, s, f(s))+K(t, s, f(s))\} d s \\
& +\int_{0}^{t}\left(\int_{0}^{s}\{H(t, s, \sigma, z(\sigma))-H(t, s, \sigma, f(\sigma))+H(t, s, \sigma, f(\sigma))\} d \sigma\right) d s \mid \\
\leq & a(t)+\int_{0}^{t} k(t, s) g(|z(s)-f(s)|) d s \\
& +\int_{0}^{t}\left(\int_{0}^{s} h(t, s, \sigma) g(|z(\sigma)-f(\sigma)|) d \sigma\right) d s
\end{aligned}
$$

Now a suitable application of the inequality in Theorem 1 part $\left(c_{2}\right)$ to (5.11) yields (5.10). 
Remark 3. We note that the inequality in Theorem 2 part $\left(d_{2}\right)$ can be used to study similar properties as in Theorems 3 and 4 for the Volterra integral equation of the form

$$
\begin{aligned}
z(x, y)= & f(x, y)+\int_{0}^{x} \int_{0}^{y} F(x, y, s, t, z(s, t)) d t d s \\
& +\int_{0}^{x} \int_{0}^{y}\left(\int_{0}^{s} \int_{0}^{t} H(x, y, s, t, \sigma, \tau, z(\sigma, \tau)) d \tau d \sigma\right) d t d s
\end{aligned}
$$

under some suitable conditions on the functions involved in equation (5.12).

In conclusion, we note that the inequalities given in Theorem 1 part $\left(c_{1}\right)$ and Theorem 2 part $\left(d_{1}\right)$ can be used respectively to study similar properties as in Theorems 3 and 4 of the equations

$$
\begin{aligned}
z(t)= & f(t)+\int_{0}^{t} e(s, z(s)) d s+\int_{0}^{t}\left(\int_{0}^{s} K(s, \tau, z(\tau)) d \tau\right) d s \\
& +\int_{0}^{t}\left(\int_{0}^{s}\left(\int_{0}^{\tau} H(s, \tau, \sigma, z(\sigma)) d \sigma\right) d \tau\right) d s
\end{aligned}
$$

and

$$
\begin{aligned}
z(x, y)= & f(x, y)+\int_{0}^{x} \int_{0}^{y} e(s, t, z(s, t)) d t d s \\
& +\int_{0}^{x} \int_{0}^{y}\left(\int_{0}^{s} \int_{0}^{t} K(s, t, \sigma, \tau, z(\sigma, \tau)) d \tau d \sigma\right) d t d s \\
& +\int_{0}^{x} \int_{0}^{y}\left(\int_{0}^{s} \int_{0}^{t}\left(\int_{0}^{\sigma} \int_{0}^{\tau} H(s, t, \sigma, \tau, m, n, z(m, n)) d n d m\right) d \tau d \sigma\right) d t d s
\end{aligned}
$$

under some suitable conditions on the functions involved in equations (5.13) and (5.14).

\section{References}

[1] D. Bainov and P. Simeonov, Integral Inequalities and Applications, Kluwer Academic Publishers, Dordrecht, 1992.

[2] E. Beckenbach and R. Bellman, Inequalities, Springer-Verlag, Berlin, New York, 1965.

[3] Ya. V. Bykov and Kh. M. Salpagarov, On the theory of integro-differential equations, in: Investigations in Integro-Differential Equations in Khirghizia 2 Izd. Akad. Nauk Kirghizia SSR, 1962 (in Russian).

[4] S. S. Dragomir, Some Gronwall Type Inequalities and Applications, Nova Science Publishers Inc., New York, 2003.

[5] A. N. Filatov and L. V. Sharova, Integral inequalities and the theory of nonlinear oscillations, Nauka, Moscow, 1976 (in Russian).

[6] D. S. Mitrinovic, J. E. Pecaric and A. M. Fink, Inequalities for Functions and their Integrals and Derivatives, Kluwer Academic Publishers, Dordrecht, 1994. 
[7] B. G. Pachpatte, Inequalities for Differential and Integral Equations, Academic Press, New York, 1998.

[8] W. Rudin, Principles of Mathematical Analysis, McGraw Hill, Book Company, New York, 1953.

57 Shri Niketan Colony, Near Abhinay Talkies, Aurangabad 431001 (Maharashtra), India.

E-mail: bgpachpatte@hotmail.com 